\title{
Weighted Vector-Valued Holomorphic Functions on Banach Spaces
}

\author{
Enrique Jordá \\ Escuela Politécnica Superior de Alcoy, IUMPA, Universitat Politècnica de València, Plaza Ferrándiz y Carbonell 1, 03801 Alcoy, Spain \\ Correspondence should be addressed to Enrique Jordá; ejorda@mat.upv.es
}

Received 11 February 2013; Accepted 14 May 2013

Academic Editor: Anna Mercaldo

Copyright (C) 2013 Enrique Jordá. This is an open access article distributed under the Creative Commons Attribution License, which permits unrestricted use, distribution, and reproduction in any medium, provided the original work is properly cited.

\begin{abstract}
We study the weighted Banach spaces of vector-valued holomorphic functions defined on an open and connected subset of a Banach space. We use linearization results on these spaces to get conditions which ensure that a function $f \operatorname{defined}$ in a subset $A$ of an open and connected subset $U$ of a Banach space $X$, with values in another Banach space $E$, and admitting certain weak extensions in a Banach space of holomorphic functions can be holomorphically extended in the corresponding Banach space of vector-valued functions.
\end{abstract}

\section{Introduction, Notation, and Preliminaries}

Let $E$ be a locally convex space. The problem of deciding when a function $f: \Omega \subset \mathbb{C} \rightarrow E$ is holomorphic whenever $u \circ f \in$ $H(\Omega)$ for each $u \in E^{\prime}$ goes back to Dunford [1], who proved that this happens when $E$ is a Banach space. Grothendieck [2] extended the result for $E$ being quasicomplete. Bogdanowicz [3] gives extension results through weak extension, that is, he proved between other results that if $\Omega_{1} \subset \Omega_{2} \subset \mathbb{C}$ are two domains (open and connected subsets), $E$ is a complex, sequentially complete, and locally convex Hausdorff space, and $f: \Omega_{1} \rightarrow E$ satisfies that $u \circ f$ admits holomorphic extension for each $u \in E^{\prime}$, then $f$ admits a holomorphic extension to $\Omega_{2}$. More recently Grosse-Erdmann, Arendt and Nikolski, Bonet, Frerick, Wengenroth, and the author have given results in this way smoothing the conditions on $\Omega_{1}$ and also requiring extensions of $u \circ f$ only for a proper subset $H \subseteq E^{\prime}$ (cf. [4-8]). Also, Laitila and Tylli have recently discussed the difference between strong and weak definitions for important spaces of vector-valued functions [9, Section 6].

Our main aim here is to analyze a weak criterion for holomorphy and to give extension results for the Banach spaces of holomorphic functions defined on a nonvoid open subset $U$ of a Banach space $X$. To obtain these extension results, we use linearization results, that is, theorems which permit to identify classes of vector valued functions defined in $U$ and with values in $E$ with continuous linear mappings from a certain space $G$ and with values in $E$. Recent work of Beltrán [10], Carando and Zalduendo [11], and Mujica [12] is devoted to get linearization results. We use for our extension results also linearization results obtained by Bierstedt in [13, 14].

Our notation for the Banach spaces, locally convex spaces, and functional analysis is standard. We refer the reader to [15-17]. For a locally convex space $F$ which is nonnormed, we denote by $F^{\prime}$ its topological dual. For a Banach space $(E,\|\cdot\|)$, the dual of $E$ is denoted by $E^{*}$. We mainly deal with Banach spaces. The absolutely convex hull of a subset $C$ of $E$ is denoted by $\Gamma(C)$, and the closure of $C$ is denoted by $\bar{C}$. If the closure is taken with respect to other topology $\tau$, it will be denoted by $\bar{C}^{\tau} .(E, w)$ and $\left(E^{*}, w *\right)$ are $E$ and $E^{*}$ endowed with the weak $\left(\sigma\left(E, E^{*}\right)\right)$ and the weak $\left(\sigma\left(E^{*}, E\right)\right)$ topology, respectively. The open unit ball of $E$ will be denoted by $B_{E}$. A subset $M \subseteq E\left(M \subseteq E^{*}\right)$ is said to be total if $\operatorname{span}(M)$ is $\left(\sigma\left(E^{*}, E\right)\right)$ dense. By the Hahn Banach theorem, $M$ being total in $E^{*}$ is equivalent to $M$ being separating, that is, if $e \in E$ and $u(e)=0$ for all $u \in M$, then $e=0 . M \subseteq E^{*}$ is said to be norming if $M$ is bounded, and its associated functional $q_{M}: E \rightarrow \mathbb{R}, e \mapsto \sup \{|u(e)|: u \in M\}$ defines an equivalent norm in $E$, that is, if the polar set $M^{\circ}:=\{e \in E:|u(e)| \leq$ 1 for all $u \in M$ \} defines an equivalent (closed) unit ball in $E$. It is immediate that if $M$ is norming then it is also separating. If $q_{M}$ is the norm of $E$, then $M$ is called 1-norming. A subset 
$M \subset E$ is called norming or total when we consider $E \subset E^{* *}$, that is, $q_{M}$ defines an equivalent norm in $E^{*}$. A subspace $H$ of $E^{*}$ is said to determine boundedness whenever all the $\sigma(E, H)$ bounded subsets of $E$ are $\left(\sigma\left(E, E^{*}\right)-\right)$ bounded. A subspace $H \subseteq E^{*}$ is said to be norming if $B_{H}$ is a norming subset of $E$. We give below a relation between these concepts. The result is given in [6, Proposition 7, Remark 8] in the more general context of the Fréchet spaces, though in this paper the norming subspaces are called almost norming. We give a proof for the Banach case because it is very transparent.

Proposition 1 (see [6]). Let E be a Banach space. A subspace $H \subset E^{*}$ is norming if and only if $\bar{H}$ determines boundedness in E.

Proof. It is standard to check that $q_{B_{H}}=q_{B_{\bar{H}}}$ on E. From this, it follows that $H$ is norming if and only if $\bar{H}$ is. Assume first that $H$ is norming. This implies that $\bar{H}$ is separating on $E$, and then, we can consider the algebraic inclusion $E \hookrightarrow \bar{H}^{*}$. By the very definition, $q_{B_{\bar{H}}}$ is the restriction of $\|\cdot\|_{\bar{H}^{*}}$ to $E$. The hypothesis $H$ norming means that $E$ is isomorphic to $\left(E, q_{B_{\bar{H}}}\right)$, which is a subspace of $\left(\bar{H}^{*},\|\cdot\|_{\bar{H}^{*}}\right)$. By the Uniform Boundedness Principle, the $\sigma(E, \bar{H})$ bounded subsets of $E$ are norm bounded in $\bar{H}^{*}$, and then, $\|\cdot\|$ bounded since the norms are supposed to be equivalent in $E$. Conversely, let one assume that $\bar{H}$ determines boundedness in $E$. This implies, again by the Uniform Boundedness Principle, that the identity $I$ : $\left(E,\|\cdot\|_{\bar{H}^{*}}\right) \rightarrow(E,\|\cdot\|)$ is bounded. Hence, there exists $C \geq 1$ such that $\|e\| \leq C\|e\|_{\bar{H}^{*}}=C q_{B_{\bar{H}}}(e)$ for each $e \in E$, which implies that $\bar{H}$ is norming.

Thus, the property of being norming for subspaces in $E^{*}$ is between weak ${ }^{*}$-dense and strongly dense.

Let $M=\left(m_{i}\right)_{i \in I}$ be a bounded subset of $E$ and $I$ an index set. Let

$$
\begin{array}{r}
l_{1}(M):=\left\{x \in E \text { : exists }\left(a_{i}\right)_{i \in I} \in l_{1}(I)\right. \\
\text { such that } \left.x=\sum a_{i} m_{i}\right\},
\end{array}
$$

equipped with the norm which makes it isomorphic to a quotient of $l_{1}(I)$. We will use the following lemma, which we supposed to be well known.

Lemma 2. Let $M \subseteq B_{E}$ be a norming subset. Then, the injection of $l_{1}(M)$ in $E$ is an onto isomorphism.

Proof. The hypothesis on $M$ yields that there exist $c, C>0$ such that

$$
c \bar{B}_{E^{*}} \subseteq M^{\circ} \subseteq C \bar{B}_{E^{*}}
$$

Hence, we take polars and apply the bipolar theorem to get

$$
\left(\frac{1}{C}\right) \bar{B}_{E} \subseteq \overline{\Gamma(M)} \subseteq\left(\frac{1}{c}\right) \bar{B}_{E}
$$

Let $C_{E}$ be the equivalent open unit ball in $E$ such that $\bar{C}_{E}=$ $\overline{\Gamma(M)}$. We define $T: l_{1}(I) \rightarrow E,\left(a_{i}\right)_{i} \mapsto \sum_{i \in I} a_{i} m_{i}$. T is clearly bounded. Moreover, $\Gamma(M) \subseteq T\left(B_{l_{1}(I)}\right)$, and then,

$$
C_{E} \subseteq \overline{\Gamma(M)} \subseteq \overline{T\left(B_{l_{1}(I)}\right)} .
$$

We get from the Schauder lemma [16, Lemma 3.9] that $T$ is open and then surjective. We conclude from the very definition of $l_{1}(M)$.

Remark 3. If we assume in Lemma 2 that $M$ is 1-norming, then the isomorphism is an isometry.

We see below that if the bounded subset $M$ is not norming, then the assertion is not true in general.

Remark 4 (Bonet). The assertion in Lemma 2 implies that if $M \subseteq E$ is bounded, then $x \in \overline{\Gamma(M)}$ if and only if for each $\varepsilon>0$ there exist sequences $\left(\alpha_{n}\right)_{n} \in(1+\varepsilon) B_{l_{1}}$ and $\left(m_{n}\right)_{n} \subset M$ such that $x=\sum_{n} \alpha_{n} m_{n}$. This is not in general true if we assume that $M$ is only to be bounded. Valdivia showed (see [17, Example 3.2.21]) that in every infinite dimensional Banach space there is an absolutely convex bounded subset $B \subset E$ which is not closed such that $E_{B}:=\left(\operatorname{span}(B), p_{B}\right)$ is a Banach space with closed unit ball $B$, where

$$
p_{B}(x):=\inf \{\lambda>0, x \in \lambda B\}, \quad x \in \operatorname{span}(B) .
$$

From $E_{B}$ being Banach, we conclude $l_{1}(B) \subseteq E_{B}$, but from the proof given in [17, Example 3.2.21], it follows that $\bar{B}$ is not included in $\operatorname{span}(B)$.

Let $U$ be a connected open subset of a Banach space $X$. The space of all the holomorphic functions on $U$ is denoted by $H(U)$. The compact open topology on $H(U)$ denoted by $\tau_{c} .\left(H(U), \tau_{c}\right)$ is a semi-Montel space; that is, each closed and bounded subset is compact. A subset $A \subset U$ is called $U$-bounded if it is bounded and the distance of $A$ to the complementary of $A$ is positive. For $U=X, U$-bounded means simply bounded. If $U$ is the unit ball of a Banach space, $A$ is $U$-bounded if and only if it is contained in a ball of radius $r<1$. If $E$ is a Banach space, the space of $E$-valued holomorphic functions on $U$ is denoted by $H(U, E)$. We refer to [18] for the precise definitions. A weight $v: U: \rightarrow] 0, \infty[$ is a continuous function which is strictly positive. According to [19], we say that a weight $v$ on $U$ satisfies the property (I) whenever it is bounded below in each $U$-bounded subset $A$ of $U$. The weighted Banach spaces of holomorphic functions are defined as

$$
\begin{aligned}
H_{v}(U):= & \left\{f \in H(U): \sup _{x \in U} v(x)|f(x)|<\infty\right\} \\
H_{v_{0}}(U):= & \{f \in H(U): v f \text { vanishes at infinity on } \\
& U \text {-bounded sets }\} .
\end{aligned}
$$

Recall that a function $g: U \rightarrow \mathbb{R}$ is said to vanish at infinity on $U$-bounded sets when for each $\varepsilon>0$ there exists a $U$ bounded subset $A$ such that $|g(x)|<\varepsilon$ for $x \in U \backslash A$. $H_{v}(U)$ 
is always continuously embedded in $\left(H(U), \tau_{c}\right)$. If $v$ satisfies $(I)$, then $H_{v}(U)$ is continuously embedded in the space of holomorphic functions of bounded type $H_{b}(U)$, which is $H(U)$ endowed with the Fréchet topology of uniform convergence on $U$-bounded sets.

Analogously, for a Banach space $E$, we define the weighted spaces of vector-valued functions as

$$
\begin{gathered}
H_{v}(U, E):=\left\{f \in H(U, E): \sup _{x \in U} v(x)\|f(x)\|<\infty\right\} \\
H_{v_{0}}(U, E):=\{f \in H(U, E): v\|f\| \text { vanishes at } \\
\text { infinity on } U \text {-bounded sets }\} .
\end{gathered}
$$

During all the work, our model spaces will be $H_{v}(U)$ and $H_{v_{0}}(U)$. But we will deal with general closed subspaces $A_{v}(U)$ of $H_{v}(U)$ and their corresponding vector-valued analogues $A_{v}(U, E)$ (which will be defined in the following section) in order to consider important subspaces as they are the spaces $\mathscr{P}\left({ }^{m} X\right)$ of homogeneous polynomials of degree $m$ and, in case of $U$ being bounded, the algebras $A(U)$ and $A_{u}(U)$ of holomorphic and bounded functions which are continuous and uniformly continuous on $\bar{U}$, respectively.

Let $A_{v}(U)$ be a subspace of $H_{v}(U)$. A subset $A \subseteq U$ is said to be a set of uniqueness for $A_{v}(U)$ if each $f \in A_{v}(U)$ which vanishes at $A$ is identically null. A set $A \subseteq X$ is said to be sampling for $A_{v}(U)$ if there exists some constant $C \geq 1$ such that, for every $f \in A_{v}(U)$,

$$
\sup _{x \in X} v(x)|f(x)| \leq C \sup _{a \in A} v(a)|f(a)| .
$$

In case $A_{v}(U)$ is an algebra the constant, $C$ can be always taken 1 and, according to Globevnik, the sampling sets are called boundaries [20-22]. If $A \subset U$ and $M_{A}=\left\{v(x) \delta_{x}: x \in\right.$ $A\} \subseteq A_{v}(U)^{*}$, it follows from the definitions that $M_{A} \subseteq B_{A_{v}^{*}}$, $A$ is sampling if and only if $M_{A}$ is norming, and $A$ is a set of uniqueness if and only if $M_{A}$ is total.

The sampling sets (as well as interpolation sets) of the weighted space $A^{-p}(\mathbb{D})$ (i.e., $H_{v}(\mathbb{D})$ for $v(z)=(1-z)^{p}$, $p>0$ ) were characterized by Seip in [23] in terms of certain densities.

\section{Banach Subspaces of $H_{v}(U)$ Which Are Dual Spaces}

Let one consider $A_{v}(U) \subseteq H_{v}(U)$ to be a subspace with compact closed unit ball for $\tau_{c}$. Notice that this condition implies that $A_{v}(U)$ is norm closed. We define the Banach space of vector-valued functions in a weak sense:

$$
A_{v}(U, E):=\left\{f: U \longrightarrow E: u \circ f \in A_{v}(U) \forall u \in E^{*}\right\} .
$$

Since weakly holomorphic functions are holomorphic and weakly bounded sets are bounded, it follows that for $A_{v}(U)=$ $H_{v}(U)$ this definition agrees with the strong definition given previously. Following the same steps as in [12, Theorem 2.1] (also in [24, Lemma 10]), we get that $A_{v}(U, E)$ can be identified with $L\left(G_{A_{v}}, E\right), G_{A_{v}}$ being the predual of $A_{v}(U)$ that exists by the Dixmier-Ng Theorem [25].

Remark 5. In [25], it is shown that $G_{A_{v}}$ consists of all the functionals $y \in A_{v}(U)^{*}$ such that $y$ restricted to $B_{A_{v}}$ is $\tau_{c}$ continuous. Let $A \subseteq U$. If we denote $M_{A}:=\left\{\delta_{x}: x \in A\right\} \subset$ $B_{G_{A_{v}}}$, we have that $M_{A}$ is separating in $A_{v}(U)$ if and only if $\operatorname{span}\left(M_{A}\right)$ is (weakly) dense if and only if $A$ is a set of uniqueness for $A_{v}(U)$. Analogously, $M_{A}$ is norming in $A_{v}(U)$ if and only if $A$ is sampling for $A_{v}(U)$.

Proposition 6. Let $A_{v}(U)$ be a subspace of $H_{v}(U)$ with $\tau_{c}$ compact closed unit ball. Then, $f \in A_{v}(U, E)$ if and only if there exists $T \in L\left(G_{A_{v}}, E\right)$ such that $f(x)=T\left(\delta_{x}\right)$. Moreover, the correspondence is an isometry.

Proof. If $T \in L\left(G_{A_{v}}, E\right)$, we define $f(x):=T\left(\delta_{x}\right)$. Since $T$ is continuous and $G_{v}^{*}=A_{v}(U)$, it follows that $u \circ f \in A_{v}(U)$ for each $u \in E^{*}$, and then, $f \in A_{v}(U, E)$ by the very definition.

Conversely, let $f \in A_{v}(U, E)$. We set $M_{U}:=\left\{v(x) \delta_{x}\right.$ : $x \in U\}$. Since $M_{U}$ is 1-norming in $A_{v}(U)$, we apply Lemma 2 and Remark 3, to get that $G_{A_{v}}=l_{1}(M)=\left\{\sum_{x \in U} \alpha_{x} v(x) \delta_{x}\right.$ : $\left.\sum_{x \in U}\left|\alpha_{x}\right|<\infty\right\}$. We see that $T\left(\delta_{x}\right):=f(x)$ defines a linear mapping on $G_{v}$. If $\sum_{x \in U} \alpha_{x} \delta_{x}=0$, then, for each $u \in E^{*}$,

$$
\begin{aligned}
\left\langle u, T\left(\sum_{x \in U} \alpha_{x} \delta_{x}\right)\right\rangle & =\left\langle u, \sum_{x \in U} \alpha_{x} f(x)\right\rangle \\
& =\left\langle u \circ f, \sum_{x \in U} \alpha_{x} \delta_{x}\right\rangle=0,
\end{aligned}
$$

and then, $T$ is well defined. Moreover, since $B_{G_{v}}=$ $\left\{\sum_{x \in U} \alpha_{x} v(x) \delta_{x}: \sum_{x \in U}\left|\alpha_{x}\right|<1\right\}$, it is easy to compute $\|T\|=$ $\sup \{v(x)\|f(x)\|: x \in U\}=\|f\|_{v}$.

Now, we are going to show that there are more natural spaces with compact unit ball for the compact open topology. To do this, we present a general result of complemented subspaces in the Fréchet spaces of analytic functions which could be of independent interest. We state it for Fréchet instead of Banach to include the important space $H_{b}(U)$. For $H_{v}(U)$ and $H_{b}(U)$ with $v$ radial and $U$ balanced, it is done by García et al. in [19, Proposition 3, Example 14].

Theorem 7. Let $F(U)$ be a Fréchet space of holomorphic functions on $U$ such that $F(U) \hookrightarrow\left(H(U), \tau_{c}\right)$ continuously. If, for $m \in \mathbb{N}, \mathscr{P}\left({ }^{m} X\right) \subset F(U)$, then $\mathscr{P}\left({ }^{m} X\right)$ endowed with its norm topology is a complemented subspace of $F(U)$. If $F(U)=$ $H_{v}(U)$, then $\overline{B_{v}} \cap \mathscr{P}\left({ }^{m} X\right)$ is compact in $\left(H(U), \tau_{c}\right)$.

Proof. Let one assume without loss of generality that $0 \in U$. For $f \in H(U)$, we denote $P_{m}^{f} \in \mathscr{P}\left({ }^{m} X\right)$ the $m$-homogeneous polynomial such that

$$
f(x)=f(0)+\sum_{k \in \mathbb{N}} P_{k}^{f}(x) .
$$


Let $\tau_{p}$ be denoted by the topology in $\mathscr{P}\left({ }^{m} X\right)$ of pointwise convergence on $\bar{B}_{X}$. The projection

$$
P_{m}:\left(H(U), \tau_{c}\right) \longrightarrow\left(P\left({ }^{m} X\right), \tau_{p}\right), \quad f \longmapsto P_{m}^{f},
$$

is continuous. We checked it. Let $f \in H(U)$, and let $\left(f_{i}\right)_{i \in I}$ be a net convergent to $f$ in $\left(H(U), \tau_{c}\right)$. Let $r>0$ such that the closed ball $D(0, r) \subset E$, and let $u \in E$ with $\|u\|=1$. For $i \in I$, we define $g_{i}(z):=f_{i}(z u) \in H^{\infty}\left(B_{r}\right), B_{r}$ being the ball with radius $r$ in $\mathbb{C}$. Let $g(z):=f(z u)$. We have that $\left(g_{i}\right)_{i \in I}$ converges to $g$ in $H^{\infty}\left(B_{r}\right)$. We conclude from the continuity of the evaluations of the derivatives in this last space and

$$
P_{m}^{f_{i}}(u)=\frac{g_{i}^{(m)}(0)}{m !} \quad m \in \mathbb{N}_{0}, i \in I .
$$

Hence, by the closed graph theorem, we get that the map $P_{m}: F(U) \rightarrow \mathscr{P}\left({ }^{m} X\right), f \mapsto P_{m}^{f}$ is continuous. Since the map is by hypothesis surjective and restricted to $\mathscr{P}\left({ }^{m} X\right)$ that is the identity, it also follows that $\mathscr{P}\left({ }^{m} X\right)$ is closed in $F(U)$. Thus, the inclusion $i:=\left.P_{m}\right|_{\mathscr{P}\left({ }^{m} X\right)}$ is an isomorphism. Hence, the inverse of the inclusion $j:=i^{-1}: \mathscr{P}\left({ }^{m} X\right) \rightarrow F(U)$ satisfies that $P_{m} \circ j$ is the identity in $\mathscr{P}\left({ }^{m} X\right)$. We apply [26, Chapter 2, Section 7, Proposition 3] to conclude that $\mathscr{P}\left({ }^{m} X\right)$ is complemented in $F(U)$.

We check now that $\overline{B_{v}} \cap \mathscr{P}\left({ }^{m} X\right)$ is compact for the topology of pointwise convergence on $U$. Let $\left(f_{i}\right)_{i \in I}$ be a net in $\mathscr{P}\left({ }^{m} X\right) \cap B_{v}$ such that it is convergent to $f \in B_{v}$ pointwise in $U$. Assume without loss of generality that $V:=U \cap B_{X}$ is nonempty. The net $\left(f_{i}\right)_{i \in I}$ is a bounded net in $\mathscr{P}\left({ }^{m} X\right)$ which is Cauchy for the topology of pointwise convergence in $V$. This topology is Hausdorff and weaker than the topology of pointwise convergence in $B_{X}$. Since $\left(\mathscr{P}\left({ }^{m} X\right),\|\cdot\|_{m}\right)$ is a dual space [18, Proposition 1.17], the topology of pointwise convergence on $B_{X}$ is relatively compact restricted to the bounded sets in $\left(\mathscr{P}\left({ }^{m} X\right),\|\cdot\|_{m}\right)$ and then agrees in the bounded sets with the topology of pointwise convergence on $V$. Moreover, $\overline{B_{v}} \cap \mathscr{P}\left({ }^{m} X\right)$ is bounded in $\left(\mathscr{P}\left({ }^{m} X\right),\|\cdot\|_{m}\right)$, and hence, we get that $\left(f_{i}\right)_{i}$ is convergent to $g \in \mathscr{P}\left({ }^{m} X\right)$ pointwise in $X$. Since $U \subseteq X$, we get $f=g$. We have proved that $\overline{B_{v}} \cap \mathscr{P}\left({ }^{m} X\right)$ is closed in $\overline{B_{v}}$ for the topology of pointwise convergence in $U$, and then it is compact.

For spaces $H_{v}(U)$ containing $\mathscr{P}\left({ }^{m} X\right)$, we have that $\left(\mathscr{P}\left({ }^{m} X\right),\|\cdot\|_{v}\right)$ is a subspace which is complemented and it is isomorphic to $\mathscr{P}\left({ }^{m} X\right)$ endowed with its natural norm $\|\cdot\|_{m}$. Moreover, $\left(\mathscr{P}\left({ }^{m} X\right),\|\cdot\|_{v}\right)$ has a compact unit ball for the topology of pointwise convergence in $U$, and hence, it is a dual Banach space because of Dixmier-Ng theorem [25]. We denote by $G_{v}^{m}$ the predual of $\left(\mathscr{P}\left({ }^{m} X\right),\|\cdot\|_{\nu}\right)$ and by $G^{m}$ the predual of $\left(\mathscr{P}\left({ }^{m} X\right),\|\cdot\|_{m}\right)$ obtained in [12, Theorem 2.4]. In $G^{m}$, the subset $M:=\left\{\delta_{x}: x \in B_{X}\right\}$ is norming and then spans a $\left(\sigma\left(G^{m}, \mathscr{P}\left({ }^{m} X\right)\right)-\right)$ dense subspace. The same applies for $M_{v}:=\left\{v(x) \delta_{x}: x \in U\right\}$ in $\left(\mathscr{P}\left({ }^{m} X\right),\|\cdot\|_{v}\right)$. Both $M$ and $M_{v}$ are formed by functionals which are linearly independent by [11, Proposition 1]. We check below that there is a natural isomorphism between $G^{m}$ and $G_{v}^{m}$.
Proposition 8. Let $v$ be a weight on $U$ such that $\mathscr{P}\left({ }^{m} X\right) \epsilon$ $H_{v}(U)$. The predual $G_{v}^{m}$ of $\left(\mathscr{P}\left({ }^{m} X\right),\|\cdot\|_{v}\right)$ is isomorphic to the predual $G^{m}$ of $\left(\mathscr{P}\left({ }^{m} X\right),\|\cdot\|_{m}\right)$ canonically, that is, there exists $T: G_{v}^{m} \rightarrow G^{m}$ such that $T\left(\delta_{x}\right)=\delta_{x}$ for each $x \in U$.

Proof. Let $M_{v}$ be as defined previously. If we define $T_{1}$ : $\operatorname{span}\left(M_{\mathrm{v}}\right) \rightarrow G^{m}$, by means of $\delta_{x} \mapsto \delta_{x}$, we have that $T$ is well defined since $\delta_{x}=\|x\|^{m} \delta_{x /\|x\|} \in \operatorname{span}(M)$, it is (weakly) continuous, and then, it can be extended to $\widehat{T}_{1}: G_{v}^{m} \rightarrow G^{m}$. If we consider now $\operatorname{span}\left(M_{v}\right)$ as a subspace of $G^{m}$ it is (weakly) dense since open sets are sets of uniqueness in $\mathscr{P}\left({ }^{m} X\right)$. The linear map $T_{2}: \operatorname{span}\left(M_{v}\right) \rightarrow G_{v}^{m}, \delta_{x} \mapsto \delta_{x}$ is again (weakly) continuous, and hence that we get, an extension $\widehat{T}_{2}: G^{m} \rightarrow$ $G_{v}^{m} . \widehat{T}_{2} \circ \widehat{T}_{1}: G_{v}^{m} \rightarrow G_{v}^{m}$ is a continuous linear mapping, and then, it is the identity since both coincide in $\operatorname{span}\left(M_{v}\right)$. Moreover, $T_{1}\left(G_{v}^{m}\right)$ has dense range in $G^{m}$. Hence, $\widehat{T}_{1}$ is an onto isomorphism by [26, Chapter 2, Section 7, Proposition 3].

From the linearization of these dual Banach subspaces of $H_{v}(U)$, one can get easily an extension of the Blaschketype result for vector-valued functions [4, Theorem 2.5] generalized in [7, Corollary 4.2]. The proof that we give is strongly based on the Banach-Steinhaus principle.

Proposition 9. Let $A_{v}(U)$ be a subspace of $H_{v}(U)$ which has a $\tau_{c}$-compact closed unit ball, let $A \subseteq U$ be a set of uniqueness for $A_{v}(U)$, and let $E$ be a Banach space. If $\left(f_{i}\right)_{i \in I}$ is a bounded net in $A_{v}(U, E)$ such that $\left(f_{i}(x)\right)_{i \in I}$ is convergent for each $x \in A$, then $\left(f_{i}\right)_{i \in I}$ is convergent to a function $f \in A_{v}(U, E)$ uniformly on the compact subsets of $U$.

Proof. Let $\left(T_{i}\right)_{i \in I}$ be the sequence of operators in $L\left(G_{A_{v}}, E\right)$ such that $f_{i}(x)=T_{i}\left(\delta_{x}\right)$ for each $x \in U$. Let $M_{v}=\left\{v(x) \delta_{x}\right.$ : $x \in U\} \subseteq G_{v}(U) . M_{v}$ is a 1-norming subset of $G_{v}$, that is,

$$
\overline{\Gamma\left(M_{v}\right)}=M_{v}^{\circ}=B_{A_{v}}^{\circ}=B_{G_{A_{v}}} .
$$

By hypothesis, there exists $C>0$ such that

$$
T_{i}\left(M_{v}\right)=\left\{v(x) f_{i}(x): x \in U\right\} \subset C B_{E} \text { for each } i \in I .
$$

Thus,

$$
T_{i}\left(B_{G_{A_{v}}}\right)=T_{i}\left(\overline{\Gamma\left(M_{v}\right)}\right) \subset \overline{\Gamma\left(T_{i}\left(M_{v}\right)\right)} \subseteq C \bar{B}_{E},
$$

for each $i \in I$. By Remark 5 , the subset $M_{A}:=\left\{\delta_{x}: x \in A\right\}$ is total in $G_{A_{v}}$. Since $\left(T_{i}\right)_{i \in I}$ is equicontinuous, the topology of pointwise convergence on $G_{A_{y}}$ coincides with the topology of pointwise convergence in $M_{A}$ by $[27,39.4(1)]$. Thus, $\left(T_{i}\right)_{i \in I}$ is pointwise convergent to $T \in L\left(G_{A_{v}}, E\right)$. The convergence is uniform on the compact subsets of $G_{A_{v}}$ by $[27,39.4(2)]$. If $K \subset U$ is compact, then $\left\{\delta_{x}: x \in K\right\}$ is compact in $G_{A_{y}}$. This follows from the observation that $U \rightarrow G_{A_{v}}, x \mapsto \delta_{x}$ is (weakly) holomorphic and then continuous.

Proposition 9 and Theorem 7 yield that the BanachSteinhaus theorem stated as in $[27,39.4(1)]$ can be extended to the space of vector-valued polynomials $P\left({ }^{m} X, E\right)$. Bochnak 
and Siciak showed [28, Theorem 2] that the uniform boundedness principle also is valid for polynomials.

The following results are extensions of those obtained in [7] by Frerick et al. for spaces of bounded holomorphic and harmonic functions on open subsets of finite-dimensional subspaces with values in locally convex spaces. Our results are valid for spaces of functions defined on an open and connected subset $U$ of a Banach space $X$, but we restrict to the case of Banach-valued functions. The proofs that we give here are simpler. The next theorem extends [7, Theorem 2.2].

Theorem 10. Let $v$ be a weight on $U$, let $A_{v}(U)$ be a subspace of $H_{v}(U)$ with $\tau_{c}$-compact closed unit ball, let $A$ be a set of uniqueness for $A_{v}(U)$, let $E$ be a Banach space, and let $H \subseteq$ $E^{*}$ be a subspace which determines boundedness in E. If $f$ : $A \rightarrow E$ is a function such that $u \circ f$ admits an extension $f_{u} \in A_{v}(U)$ for each $u \in H$, then $f$ admits a unique extension $F \in A_{v}(U, E)$.

Proof. Let $F_{A}$ be the span of $\left\{\delta_{x}: x \in A\right\}$. The hypothesis implies that $F_{A}$ is $\sigma\left(G_{A_{v}}, A_{v}(U)\right)$ dense, and then, it is dense in norm. The map $T: F_{A} \rightarrow E, \delta_{x} \mapsto f(x)$ is well defined since $H$ is separating. Let $x=\sum_{i=1}^{k} \alpha_{i} \delta_{x_{i}}$ be an element in the unit ball $B_{F_{A}}$ of $F_{A}$, and let $u \in H$. We compute:

$$
\begin{aligned}
|\langle T(x), u\rangle| & =\left|\left\langle\sum_{i=1}^{k} \alpha_{i} f\left(x_{i}\right), u\right\rangle\right| \\
& =\left|\left\langle\sum_{i=1}^{k} \alpha_{i} \delta_{x_{i}}, f_{u}\right\rangle\right| \leq\left\|f_{u}\right\|_{v} .
\end{aligned}
$$

Since this is true for each $x \in B_{F_{A}}$, we conclude that $T\left(B_{F_{A}}\right)$ is $\sigma(E, H)$ bounded and then norm bounded by hypothesis. Thus, $T: F_{A} \rightarrow E$ is a bounded linear mapping. Since $F_{A}$ is dense in $G_{A_{v}}$, we can extend $T$ to $\widehat{T}: G_{A_{v}} \rightarrow E$. We conclude by Proposition 6.

The following result is a generalization of [6, Theorem $1(\mathrm{ii})]$.

Theorem 11. Let $v$ be a weight on $U$, let $A_{v}(U)$ be a subspace of $H_{v}(U)$ with $\tau_{c}$-compact closed unit ball, let $A$ be a set of uniqueness for $A_{v}(U)$, let $E$ be a Banach space, and let $H \subseteq E^{*}$ be a norming subspace. If $f: A \rightarrow E$ is a function such that $u \circ f$ admits an extension $f_{u} \in A_{v}(U)$ for each $u \in H$ such that $\left(f_{u}\right)_{u \in B_{H}}$ is bounded in $A_{v}(U)$, then $f$ admits a unique extension $F \in A_{v}(U, E)$.

Proof. If $u \in B_{\bar{H}}$ and $\left(u_{n}\right)_{n} \subset B_{H}$ tend to $u$, then $\left(f_{u_{n}}\right)_{n}$ is a bounded sequence such that $\left(f_{u_{n}}(x)\right)_{n}$ converges to $u(f(x))$ for each $x \in A$. Proposition 9 yields that there exists $f_{u} \in$ $A_{v}(U)$ such that $\left(f_{u_{n}}(x)\right)_{n}$ tends to $f_{u}(x)$ for each $x \in U$. The conclusion is a consequence of Proposition 1 and Theorem 10.

We now study the problem of extending functions which admit extensions for functionals in a subspace $H$ of $E^{*}$ which we assume only to be $\sigma\left(E^{*}, E\right)$ dense. In this case, we require that $A$ is quite large. This is symmetric with the problem studied by Gramsch [29], Grosse-Erdmann [8], and
Bonet et al. [5]. The next theorem is an extension to our context of [7, Theorem 3.2].

Theorem 12. Let $v$ be a weight on $U$, let $A_{v}(U)$ be a subspace of $H_{v}(U)$ with $\tau_{c}$-compact unit ball, and let $A$ be a sampling set for $A_{v}(U)$. Let $E$ be a Banach space, and let $H$ be a $\sigma\left(E^{*}, E\right)$ dense subspace of $E^{*}$. If $f: A \rightarrow E$ is a function such $\sup _{a \in A} v(a)\|f(a)\|<\infty$ and such that $u \circ f$ admits an extension $f_{u} \in A_{v}(U)$ for each $u \in H$, then there exists a unique extension $F \in A_{v}(U, E)$ of $f$.

Proof. The set $M_{A}:=\left\{v(x) \delta_{x}: x \in A\right\} \subseteq G_{A_{v}}$ is norming for $A_{v}(U)$; hence, we apply Lemma 2 to get that $G_{A_{v}}$ is isomorphic to $l_{1}\left(M_{A}\right)$. This means that for each $x \in G_{A_{v}}$, there exists $\alpha:=\left(\alpha_{n}\right)_{n} \in l_{1}$ and $\left(x_{n}\right)_{n} \subset A$ such that

$$
x=\sum_{n} \alpha_{n} v\left(x_{n}\right) \delta_{x_{n}}
$$

The open unit ball $B_{1}$ in $G_{A_{v}}$ for the norm which makes this space isometric to $l_{1}\left(M_{A}\right)$ is formed by the vectors $x \in G_{A_{v}}$ such that the sequence $\left(\alpha_{n}\right)_{n}$ in the previous representation can be taken in the open unit ball of $l_{1}$. We define that $T$ : $G_{A_{v}} \rightarrow E, x \mapsto \sum_{n} \alpha_{n} v\left(x_{n}\right) f\left(x_{n}\right)$. Since $\{v(a) f(a): a \in A\}$ is bounded by hypothesis, the series is convergent. Moreover, if $\sum_{n} \alpha_{n} v\left(x_{n}\right) \delta_{x_{n}}=0$, then for each $u \in H$

$$
u\left(\sum_{n} \alpha_{n} v\left(x_{n}\right) f\left(x_{n}\right)\right)=\left\langle\sum_{n} \alpha_{n} v\left(x_{n}\right) \delta_{x_{n}}, f_{u}\right\rangle=0 .
$$

Since $H$ is separating, $T$ is well defined. Moreover, the hypothesis of boundedness of $\{v(a) f(a): a \in A\}$ implies that $T\left(B_{1}\right)$ is bounded. Hence, we conclude by Proposition 6 .

Remark 13. If we consider $f: B(0,1 / 2) \rightarrow l_{1}, z \rightarrow\left(z^{n}\right)_{n}$, we have that $f \in H\left(\mathbb{D}, l_{1}\right)$; hence, $f(B(0,1 / 2))$ is relatively compact in $l_{1}$. Moreover, it is immediate that $u \circ f$ admits an extension to $H^{\infty}(\mathbb{D})$ for each $u \in \varphi$ (the space of sequences which are zero but finitely many components), $\varphi$ is $\sigma\left(l_{\infty}, l_{1}\right)$ dense (even norming since it is dense in $c_{0}$ ), and $B(0,1 / 2)$ is a set of uniqueness for $H^{\infty}(\mathbb{D})$. However, $f \notin H^{\infty}\left(\mathbb{D}, l_{1}\right)$ since $\|f(z)\|_{1}=|z| /(1-|z|)$ for each $z \in \mathbb{D}$. This shows that the hypothesis in Theorems 10 and 12 is optimal, that is, for the conditions on the set $A$ where the functions are defined and in the subspace $H$ for which functionals, we have weak extensions that cannot be simultaneously relaxed, and also the condition of boundedness in the extensions inTheorem 11 can not be dropped.

\section{General Banach Subspaces of $H_{v}(U)$}

For arbitrary Banach spaces $A_{v}(U) \subset H_{v}(U)$ with no assumption on the unit ball, the equivalence between the weak and the strong definitions does not hold in general. We discuss it below. We consider the space $H_{v_{0}}(U)$, and we define

$$
H_{v_{0}}(U, E)_{w}:=\left\{f: U \longrightarrow E: u \circ f \in H_{v_{0}}(U) \forall u \in E^{*}\right\} .
$$


A Banach space $E$ is said to satisfy the Schur property if every sequence $\left(x_{n}\right)_{n}$ in $E$ which is weakly convergent is also norm convergent. The well-known theorem of Schur asserts that $l_{1}$ satisfies this property.

Proposition 14. If $E$ is a Banach space with the Schur property, then $H_{v_{0}}(U, E)=H_{v_{0}}(U, E)_{w}$.

Proof. Suppose that there exists $f \in H_{v_{0}}(U, E)_{w} \backslash H_{v_{0}}(U, E)$. Then, there exist $c>0$ and $\left(x_{n}\right)_{n}$ going to infinity on $U$ bounded sets such that $v\left(x_{n}\right)\left\|f\left(x_{n}\right)\right\|>c$ and $u \circ f \in H_{v_{0}}(U)$ for all $u \in E^{*}$. This last condition implies that

$$
\left\{v\left(x_{n}\right) f\left(x_{n}\right): n \in \mathbb{N}\right\}
$$

is (weakly) convergent to zero, a contradiction.

We see below that the situation differs for function with values in the general Banach spaces.

Example 15. Assume that $X$ is finite dimensional and $H_{v_{0}}(U)$ is infinite dimensional. Then, $H_{v_{0}}\left(U, c_{0}\right) \subsetneq H_{v_{0}}\left(U, c_{0}\right)_{w}$.

Proof. First, we proceed similarly as in [30, Lemma 21] to get a sequence $\left(f_{n}\right)_{n}$ in $B_{v_{0}}$ such that $\left(f_{n}\right)_{n}$ converges to 0 in $\tau_{c}$, and there exists $\delta>0$ such that $\left\|f_{n}\right\|_{v} \geq \delta$ for all $n \in \mathbb{N}$. Since $H_{v_{0}}(U)$ is infinite dimensional, there is $\delta>0$ and $\left(g_{n}\right)_{n} \in B_{v_{0}}$ such that $\left\|g_{n}-g_{k}\right\|_{v}>\delta$ for $n \neq k$. We apply that $\left(H_{v}(U), \tau_{c}\right)$ is metrizable and $\overline{B_{v}}$ is $\tau_{c}$ compact to get that $\left(B_{v_{0}}, \tau_{c}\right)$ is relatively sequentially compact. Hence, we can extract a subsequence of $\left(g_{n}\right)_{n}$ which is Cauchy for $\tau_{c}$, and we denote again by $\left(g_{n}\right)_{n}$. Defining $f_{n}:=\left(g_{n}-g_{n+1}\right) / 2$, we get the desired sequence.

We consider $f: U \rightarrow c_{0}, z \rightarrow\left(f_{n}(z)\right)_{n}$. Let $u=\left(u_{n}\right)_{n} \in l_{1}$ be arbitrary. Since $\left(f_{n}\right)_{n} \subset B_{v_{0}}$, the series $\sum_{n} u_{n} f_{n}$ is convergent in $H_{v_{0}}(U)$. Hence, $f \in H_{v_{0}}\left(U, c_{0}\right)_{w}$. The convergence of $\left(f_{n}\right)_{n}$ for the compact open topology implies that for each $K \subset U$ there exists $n_{0}=n_{0}(K)$ such that

$$
\sup \left\{\left|f_{n_{0}}(z)\right|: z \in K\right\}<\frac{\delta}{2 \max \{v(x): x \in K\}} .
$$

Since $\left\|f_{n_{0}}\right\|_{v} \geq \delta$, we obtain that there exists $z_{0} \in U \backslash K$ such that

$$
v\left(z_{0}\right)\left\|f\left(z_{0}\right)\right\| \geq v\left(z_{0}\right)\left|f_{n_{0}}\left(z_{0}\right)\right| \geq \frac{\delta}{2} .
$$

Thus, $f \notin H_{v_{0}}\left(U, c_{0}\right)$.

Example 16. Assume that $U$ is the unit ball of a Banach space $X, g:[0,1] \rightarrow] 0, \infty[$ continuous with $g(t)>0$ for $0 \leq$ $t<1$ and $g(1)=0$ and $v(x)=g(\|x\|)$ for $x \in U$. Then, $H_{v_{0}}\left(U, c_{0}\right)_{w} \subsetneq H_{v_{0}}\left(U, c_{0}\right)$.

Proof. The hypothesis on $v$ implies that for each $f \in H_{v}$ the Taylor polynomials $P_{n}^{f}$ of the development at zero converge to $f$ in $\left(H(U), \tau_{b}\right)$. If we consider the Cesàro means

$$
C_{n}(f)=\frac{1}{n+1} \sum_{i=0}^{n}\left(\sum_{k=0}^{i} P_{n}(f)\right),
$$

then $C_{n}(f) \in B_{v_{0}}$ for each $f \in B_{v}$ ([31, Proposition 1.2], [19, Proposition 4]) and $C_{n}(f) \rightarrow f$ in $\left(H(U), \tau_{b}\right)$. If $f \in B_{v} \backslash B_{v_{0}}$, then $\left(C_{n}(f)\right)_{n}$ is not Cauchy in $H_{v}(U)$, since $H_{v_{0}}(U)$ is closed. Hence, there are $\varepsilon>0$ and a subsequence $\left(g_{k}\right)_{k}:=\left(C_{n_{k}}(f)\right)_{k}$ such that

$$
\left\|g_{k}-g_{k+1}\right\|_{v}>\varepsilon
$$

Defining $h_{k}:=g_{k}-g_{k+1}$, we have that $\left(h_{k}\right)_{k}$ tends to 0 in $H_{b}(U)$. Proceeding as in Example 15, we obtain that $h: U \rightarrow$ $c_{0}, x \mapsto\left(h_{k}(x)\right)_{k}$ satisfies $h \in H_{v_{0}}\left(U, c_{0}\right)_{w} \backslash H_{v_{0}}\left(U, c_{0}\right)$.

The proof is complete since $H_{v}(U) \backslash H_{v_{0}}(U)$ is never empty. We checked it. If $U=\mathbb{D}$, then $H_{v}(\mathbb{D})$ is the bidual of $H_{v_{0}}(\mathbb{D})$ and this last space is not reflexive [32,33]. Hence, there exists $f_{0} \in H_{v}(\mathbb{D}) \backslash H_{v_{0}}(\mathbb{D})$ with $\left\|f_{0}\right\|_{v} \leq 1$. For $U$ arbitrary, we consider $x_{0} \in X$ such that $\left\|x_{0}\right\|=1$ and $u \in X^{*}$ such that $\|u\|=1$ and $u\left(x_{0}\right)=1$. Define $f: U \rightarrow \mathbb{C}$ by $f(x)=f_{0}(u(x))$. Since $g$ is nonincreasing, we have

$$
\begin{aligned}
v(x)|f(x)| & =g(\|x\|)\left|f_{0}(u(x))\right| \\
& \leq g(|u(x)|)\left|f_{0}(u(x))\right| \leq 1 .
\end{aligned}
$$

Since $f_{0} \notin H_{v_{0}}(\mathbb{D})$, there exist $c>0$ and a sequence $\left(z_{n}\right)_{n}$ of complex numbers smaller than 1 such that $\lim _{n}\left|z_{n}\right|=1$ and

$$
v\left(z_{n} x_{0}\right)\left|f\left(z_{n} x_{0}\right)\right|=g\left(\left|z_{n}\right|\right)\left|f_{0}\left(z_{n}\right)\right|>c,
$$

hence, $f \notin H_{v_{0}}(U)$.

Thus, on the contrary that with the concrete examples of dual spaces $A_{v}(U)$ considered in the previous section $\left(H_{v}(U)\right.$ and $\left.\mathscr{P}\left({ }^{m} X\right)\right)$, in $H_{v_{0}}(U)$ the definition of the corresponding spaces of vector-valued functions in the weak sense are not consistent with the natural definition. For linearization for these spaces with the weak definition, we refer to the work of Carando and Zalduendo [11].

In view of Proposition 14, one could expect that the analogous extensions of Theorems 10 and 12 are possible for $H_{v_{0}}(U, E)$ when $E$ is required to have the Schur property. This is not the case as the following example shows.

Example 17. Let $U$ be the unit ball of a Banach space $X$, and let $v(x)=1-\|x\|$ for $x \in U$. Fix $x_{0} \in X$ with $\left\|x_{0}\right\|=1$ and $h \in B_{X^{*}}$ with $h\left(x_{0}\right)=1$. Consider that $f: U: \rightarrow l_{1}$, $x \mapsto\left(h(x)^{n}\right)_{n}$, then the following applies.

(a) $f \in H_{v}\left(U, l_{1}\right)$ and for each $0<r<1$

$$
\|f\|_{v}=\sup _{x \in U \backslash r U} v(x)\|f(x)\|_{1}=1 .
$$

Hence, $f \notin H_{v_{0}}\left(U, l_{1}\right)$.

(b) $u \circ f \in H_{v_{0}}(U)$ for each $u=\left(u_{n}\right)_{n} \in c_{0}$. 
Proof. To prove (a), we observe that $p(t)=t /(1-t)$ is increasing for $t \in[0,1[$; hence,

$$
\begin{aligned}
\sup _{x \in U} v(x)\|f\|_{1} & =\sup _{x \in U}(1-\|x\|) \frac{|h(x)|}{1-|h(x)|} \\
& =\sup _{x=t x_{0}, 0<t<1}(1-\|x\|) \frac{|h(x)|}{1-|h(x)|} \\
& =\sup _{x=t x_{0}, 0<t<1}|h(x)| \\
& =\sup _{x=t x_{0}, r<t<1}(1-\|x\|) \frac{|h(x)|}{1-|h(x)|}=1 .
\end{aligned}
$$

Let $u=\left(u_{n}\right)_{n} \in c_{0}$. Let $\varepsilon>0$ and $n_{0} \in \mathbb{N}$ such that $n>n_{0}$ implies $\left|u_{n}\right|<\varepsilon / 2$. For each $x \in U$, since $|h(x)|<1$, we have

$$
v(x)\left|\sum_{n>n_{0}} u_{n} h(x)^{n}\right| \leq(1-\|x\|) \frac{\varepsilon}{2} \sum_{n \in \mathbb{N}}|h(x)|^{n} \leq \frac{\varepsilon}{2} .
$$

Let $0<r<1$ such that for each $r<t<1$

$$
(1-t) \sum_{n \leq n_{0}}\left|u_{n}\right|<\frac{\varepsilon}{2} \text {. }
$$

From (30), (31), and $|h(x)|<1$, we obtain that

$$
\sup _{x \in U \backslash r U} v(x)|u \circ f(x)|=\sup _{x \in U \backslash r U}(1-\|x\|) \sum_{n \in \mathbb{N}}\left|u_{n} h(x)^{n}\right| \leq \varepsilon .
$$

Remark 18. The same computation as in Example 17(b) shows that for $1<p<\infty$ and $v(x)=(1-\|x\|)^{(1 / p)}$ the function $f: U \rightarrow l_{p}, x \mapsto\left(h(x)^{n}\right)_{n}$ satisfies that $f \in H_{v_{0}}\left(U, l_{p}\right)_{w} \backslash$ $H_{v_{0}}\left(U, l_{p}\right)$.

\section{Spaces of Weighted Compact Range Vector-Valued Holomorphic Functions}

In this section, we consider the natural extension to the weighted case of the vector-valued compact holomorphic functions introduced by Aron and Schottenloher in [34] by means of the weak definition, that is, for an open and connected subset $U$ of a Banach space $X$, a closed subspace $A_{v}(U)$ of $H_{v}(U)$, and a Banach space $E$, we define that

$$
\begin{gathered}
A_{v}^{c}(U, E)=\left\{f \in H_{v}(U, E):(v f)(U)\right. \text { is relatively compact } \\
\text { and } \left.u \circ f \in A_{v}(U) \forall u \in E^{*}\right\} .
\end{gathered}
$$

In case $X$ is finite dimensional, the space $H_{v_{0}}(U, E)$ is the space of holomorphic functions such that $f$ is continuous in the Alexandroff compactification $U \cup\{\infty\}$ of $U$ and $f(\infty)=$ 0 . Hence, $H_{v_{0}}(U, E)=H_{v_{0}}^{c}(U, E)$ in this case. If $X$ is infinite dimensional, the inclusion $H_{v_{0}}^{c}(U, E) \subset H_{v_{0}}(U, E)$ is strict in general. Observe that if $U$ is the unit ball and $v$ vanishes at $\infty$ on $U$, then $\left.I\right|_{U} \in H_{v_{0}}(U, X)$.

We check that this (weak) definition agrees with the natural definition when $U$ is the unit ball of $X, v=1$, and $A_{v}(U)=A_{u}(U)$ the space of the holomorphic and uniformly continuous functions on $U$, that is, we want to show that

$$
\begin{gathered}
A_{u}^{c}(U, E):=\{f \in H(U, E): f \text { is uniformly continuous } \\
\text { and } f(U) \text { is relatively compact }\} \\
=\left\{f: U \longrightarrow E: u \circ f \in A_{u}(U)\right. \\
\text { for each } u \in E^{*} \text { and }
\end{gathered}
$$$$
f(U) \text { is relatively compact }\} \text {. }
$$

Assume that $f \in H(U, E)$ satisfies that $u \circ f \in A_{u}(U)$ for each $u \in E^{*}$. Given $\varepsilon>0$, since $f(U)$ is relatively compact, there exists a weak neighbourhood $V$ of 0 such that

$$
(f(U)-f(U)) \cap V \subseteq(f(U)-f(U)) \cap B(0, \varepsilon) .
$$

Let one assume that $V:=\left\{e \in E:\left|u_{i}(e)\right|<\gamma, 1 \leq i \leq k\right\}$. Since $u_{i} \circ f$ is uniformly continuous on $U$ for $1 \leq i \leq k$, there exists $\delta>0$ such that $\|x-y\|<\delta$ implies $f(x)-f(y) \in V$, and therefore, $\|f(x)-f(y)\|<\varepsilon$.

Given two locally convex spaces $F$ and $E$, we denote by $F \varepsilon E$ its $\varepsilon$-product of Schwartz, that is, the space of all linear and continuous mappings $\mathscr{L}_{e}\left(F_{\text {co }}^{\prime}, E\right)$, endowed with the topology of uniform convergence on the equicontinuous subsets of $F^{\prime} . F_{\text {co }}^{\prime}$ is $F^{\prime}$ endowed with the topology $\tau_{\text {co }}$ of uniform convergence on the convex compact subsets of $F$. The $\varepsilon$ product is symmetric by means of the transpose mapping [27, 43.3(3)]. In case $E$ and $F$ being Banach spaces, $T: F^{*} \rightarrow E$ belongs to $F \varepsilon E$ if and only if $T$ is a compact operator which is weak $^{*}$-weak continuous by $[27,43.3(2)]$. The next theorem is the analogous of Theorem 12 in the case of general Banach spaces of functions, not necessarily dual Banach spaces. However, the techniques used here are different. The proof is analogous to the one given by Bierstedt and Holtmanns in [35] when the linearization result is obtained in a much more general context, but we only require the function to be defined in a sampling set.

Theorem 19. Let $A_{v}(U)$ be a closed subspace of $H_{v}(U)$, and let $A \subset U$ be a sampling set for $H_{v}(U)$. Let $E$ be a Banach space, and let $H$ be a weak ${ }^{*}$-dense subspace of $E^{*}$. The following are equivalent.

(i) $f: A \rightarrow$ E satisfies that $(v f)(A)$ is relatively compact in $E$, and $u \circ f$ admits an extension $f_{u} \in A_{v}(U)$ for each $u \in H$.

(ii) The linear mapping $T: H \rightarrow A_{v}(U), u \rightarrow f_{u}$ admits an extension $\widehat{T} \in A_{v}(U) \varepsilon E$.

(iii) $f$ can be extended to $F \in A_{v}^{c}(U, E)$.

Proof. If $f$ satisfies (i), then the linear mapping $T: H \rightarrow$ $A_{v}(U), u \mapsto f_{u}$ is $\tau_{\text {co }}-\|\cdot\|$ continuous, since the absolute 
convex hull of $f(A)$ is relatively compact in $E$, and the uniform convergence on $A$ defines an equivalent topology in $A_{v}(U)$. By the Hahn-Banach theorem, $H$ is dense in $E^{*}$ endowed with any topology $t$ such that $E=\left(E^{*}, \sigma\left(E^{*}, E\right)\right)^{\prime}=$ $\left(E^{*}, t\right)^{\prime}$, that is, which respects the duality of $E^{*}$ and $E$, in particular, for $E_{\mathrm{co}}^{*}:=\left(E^{*}, \tau_{\mathrm{co}}\right)$. Since $A_{v}(U)$ is a Banach space, we can extend it to $\widehat{T} \in \mathscr{L}\left(E_{\mathrm{co}}^{*}, A_{v}(U)\right)=E \varepsilon A_{v}(U)$.

If (ii) is satisfied, then the transpose $\widehat{T}^{t}: A_{v}(U)^{*} \rightarrow E$ is weak ${ }^{*}$-weak continuous, and $\widehat{T}^{t}$ maps the unit ball of $A_{v}(U)^{*}$ to a relatively compact subset of $E([27,43.3(2),(3)])$. We define $f(x)=\widehat{T}^{t}\left(\delta_{x}\right)$, since $\left.u \circ f(x)=\left\langle T(u), \delta_{x}\right)\right\rangle$ for each $x \in U$, and for each $u \in E^{*}$, we have that $u \circ f \in A_{v}(U)$. We conclude since $\left\{v(x) \delta_{x}: x \in U\right\}$ is in the unit ball of $A_{v}(U)^{*}$.

Finally, that (iii) implies (i) is trivial.

Observe that, setting $A=U$ in Theorem 19, we obtain a linearization of the space $A_{v}^{c}(U, E)$, which also can be obtained as a consequence of the much more general linearization result given by Bierstedt in [14, Bemerkung 3.1] and [15, Corollary 3.94]. Example 15 shows that Theorems 10 and 12 cannot be stated avoiding the condition of relative compactness on the range for general Banach spaces of holomorphic functions.

We finish showing that the weak definition given in this section for $H_{v_{0}}^{c}(U, E)$ is consistent with the natural one, that is,

$$
\begin{aligned}
H_{v_{0}}^{c}(U, E)=\{ & f f \in H_{v_{0}}(U, E): \\
& (v f)(U) \text { is relatively compact }\} \\
= & \left\{f \in H_{v}(U, E):\right. \\
& (v f)(U) \text { is relatively compact and } \\
& \left.u \circ f \in H_{v_{0}}(U) \forall u \in E^{*}\right\} .
\end{aligned}
$$

We use a similar argument to the one used by Bierstedt in [13, page 200] in a more general setting, including our case when $X$ is finite dimensional (i.e., putting compact instead of $U$-bounded in the definition of $\left.H_{v_{0}}(U)\right)$. If $f: U \rightarrow E$ satisfies that $u \circ f \in H_{v_{0}}(U)$ for each $u \in E^{*}$, then by the previous theorem, there exists $T: H_{v_{0}}(U)^{*} \rightarrow E$ defined by $T\left(\delta_{x}\right)=f(x)$ which is weak ${ }^{*}$-weak continuous and such that $T\left(B_{v_{0}}\right)$ is relatively compact. This implies that the restriction of $T$ to $B_{v_{0}}$ is weak ${ }^{*}$-norm continuous. Let $\varepsilon>0$. There exists a weak ${ }^{*} 0$-neighbourhood $V$ in $H_{v_{0}}(U)^{*}$ such that $\|T(y)\|<$ $\varepsilon$ for every $y \in V$. Let $\left\{f_{1}, \ldots, f_{k}\right\} \subseteq H_{v_{0}}(U)$ be such that $V=\left\{u \in H_{v_{0}}(U)^{*}:\left|u\left(f_{i}\right)\right|<1,1 \leq i \leq k\right\}$. There exists a $U$ bounded subset $K$ such that $v(x)\left|f_{i}(x)\right| \leq 1$ for each $x \in U \backslash K$. This yields that $\left\{v(x) \delta_{x}: x \in U \backslash K\right\} \in V$, and consequently, $v(x)\|f(x)\|=\left\|T\left(v(x) \delta_{x}\right)\right\| \leq \varepsilon$ for every $x \in U \backslash K$.

\section{Acknowledgments}

The author wants to thank J. Bonet for several references, discussions, and ideas provided, which were very helpful and in particular allowed him to prove Theorem 7, Proposition 8 , and Examples 15 and 16. Remark 4 is due to him. The participation of M. J. Beltrán in a lot of discussions during all the work has also been very important. Her ideas are also reflected in the paper. The author is also indebted to L. Frerick and J. Wengenroth for communicating to him Lemma 2. The remarks and corrections of the referee have been also really helpful to the final version. The author thanks him/her for that. This research was partially supported by MEC and FEDER Project MTM2010-15200, GV Project ACOMP/2012/090, and Programa de Apoyo a la Investigacin y Desarrollo de la UPV PAID-06-12.

\section{References}

[1] N. Dunford, "Uniformity in linear spaces," Transactions of the American Mathematical Society, vol. 44, no. 2, pp. 305-356, 1938.

[2] A. Grothendieck, "Sur certains espaces de fonctions holomorphes. I," Journal für die Reine und Angewandte Mathematik, vol. 192, pp. 35-64, 1953.

[3] W. M. Bogdanowicz, "Analytic continuation of holomorphic functions with values in a locally convex space," Proceedings of the American Mathematical Society, vol. 22, pp. 660-666, 1969.

[4] W. Arendt and N. Nikolski, "Vector-valued holomorphic functions revisited," Mathematische Zeitschrift, vol. 234, no. 4, pp. 777-805, 2000.

[5] J. Bonet, L. Frerick, and E. Jordá, "Extension of vector-valued holomorphic and harmonic functions," Studia Mathematica, vol. 183, no. 3, pp. 225-248, 2007.

[6] L. Frerick and E. Jordá, "Extension of vector-valued functions," Bulletin of the Belgian Mathematical Society. Simon Stevin, vol. 14, no. 3, pp. 499-507, 2007.

[7] L. Frerick, E. Jordá, and J. Wengenroth, "Extension of bounded vector-valued functions," Mathematische Nachrichten, vol. 282, no. 5, pp. 690-696, 2009.

[8] K.-G. Grosse-Erdmann, "A weak criterion for vector-valued holomorphy," Mathematical Proceedings of the Cambridge Philosophical Society, vol. 136, no. 2, pp. 399-411, 2004.

[9] J. Laitila and H.-O. Tylli, "Composition operators on vectorvalued harmonic functions and Cauchy transforms," Indiana University Mathematics Journal, vol. 55, no. 2, pp. 719-746, 2006.

[10] M. J. Beltrán, "Linearization of weighted (LB)-spaces of entire functions on Banach spaces," Revista de la Real Academia de Ciencias Exactas, Físicas y Naturales A, vol. 106, no. 2, pp. 275286, 2012.

[11] D. Carando and I. Zalduendo, "Linearization of functions," Mathematische Annalen, vol. 328, no. 4, pp. 683-700, 2004.

[12] J. Mujica, "Linearization of bounded holomorphic mappings on Banach spaces," Transactions of the American Mathematical Society, vol. 324, no. 2, pp. 867-887, 1991.

[13] K.-D. Bierstedt, "Gewichtete Räume stetiger vektorwertiger Funktionen und das injektive Tensorprodukt. I," Journal für die Reine und Angewandte Mathematik, vol. 259, pp. 186-210, 1973.

[14] K.-D. Bierstedt, "Gewichtete Räume stetiger vektorwertiger Funktionen und das injektive Tensorprodukt. II," Journal für die Reine und Angewandte Mathematik, vol. 260, pp. 133-146, 1973.

[15] M. Fabian, P. Habala, P. Hájek, V. Montesinos, and V. Zizler, Banach Space Theory, Springer, New York, NY, USA, 2011.

[16] R. Meise and D. Vogt, Introduction to Functional Analysis, vol. 2 of Oxford Graduate Texts in Mathematics, The Clarendon Press Oxford University Press, New York, NY, USA, 1997, Translated 
from the German by M. S. Ramanujan and revised by the authors.

[17] P. Pérez Carreras and J. Bonet, Barrelled Locally Convex Spaces, North-Holland Mathematics Studies 131, Notas de Matemtica [Mathematical Notes] 113, North-Holland, Amsterdam, The Netherlands, 1987.

[18] S. Dineen, Complex Analysis on Infinite-Dimensional Spaces, Springer Monographs in Mathematics, Springer, London, UK, 1999.

[19] D. García, M. Maestre, and P. Rueda, "Weighted spaces of holomorphic functions on Banach spaces," Studia Mathematica, vol. 138, no. 1, pp. 1-24, 2000.

[20] C. Boyd and S. Lassalle, "Geometry and analytic boundaries of Marcinkiewicz sequence spaces," The Quarterly Journal of Mathematics, vol. 61, no. 2, pp. 183-197, 2010.

[21] J. Globevnik, "On interpolation by analytic maps in infinite dimensions," Mathematical Proceedings of the Cambridge Philosophical Society, vol. 83, no. 2, pp. 243-252, 1978.

[22] J. Globevnik, "Boundaries for polydisc algebras in infinite dimensions," Mathematical Proceedings of the Cambridge Philosophical Society, vol. 85, no. 2, pp. 291-303, 1979.

[23] K. Seip, "Beurling type density theorems in the unit disk," Inventiones Mathematicae, vol. 113, no. 1, pp. 21-39, 1993.

[24] J. Bonet, P. Domański, and M. Lindström, "Weakly compact composition operators on analytic vector-valued function spaces," Annales Academice Scientiarum Fennica, vol. 26, no. 1, pp. 233-248, 2001.

[25] K. F. Ng, "On a theorem of Dixmier," Mathematica Scandinavica, vol. 29, pp. 279-280, 1971.

[26] J. Horváth, Topological Vector Spaces and Distributions. Vol. I, Addison-Wesley, London, UK, 1966.

[27] G. Köthe, Topological Vector Spaces. II, vol. 237, Springer, Berlin, Germany, 1979.

[28] J. Bochnak and J. Siciak, "Polynomials and multilinear mappings in topological vector spaces," Studia Mathematica, vol. 39, pp. 59-76, 1971.

[29] B. Gramsch, "Ein Schwach-Stark-Prinzip der Dualitätstheorie lokalkonvexer Räume als Fortsetzungsmethode," Mathematische Zeitschrift, vol. 156, no. 3, pp. 217-230, 1977.

[30] J. Bonet, M. C. Gómez-Collado, D. Jornet, and E. Wolf, "Operator-weighted composition operators between weighted spaces of vector-valued analytic functions," Annales Academice Scientiarum Fennicee, vol. 37, no. 2, pp. 319-338, 2012.

[31] K. D. Bierstedt, J. Bonet, and A. Galbis, "Weighted spaces of holomorphic functions on balanced domains," The Michigan Mathematical Journal, vol. 40, no. 2, pp. 271-297, 1993.

[32] K. D. Bierstedt and W. H. Summers, "Biduals of weighted Banach spaces of analytic functions," Journal of the Australian Mathematical Society A, vol. 54, no. 1, pp. 70-79, 1993.

[33] J. Bonet and E. Wolf, "A note on weighted Banach spaces of holomorphic functions," Archiv der Mathematik, vol. 81, no. 6, pp. 650-654, 2003.

[34] R. M. Aron and M. Schottenloher, "Compact holomorphic mappings on Banach spaces and the approximation property," Bulletin of the American Mathematical Society, vol. 80, pp. 12451249, 1974.

[35] K. D. Bierstedt and S. Holtmanns, "Weak holomorphy and other weak properties," Bulletin de la Société Royale des Sciences de Liège, vol. 72, no. 6, pp. 377-381, 2004. 


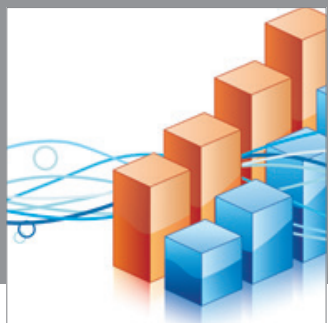

Advances in

Operations Research

mansans

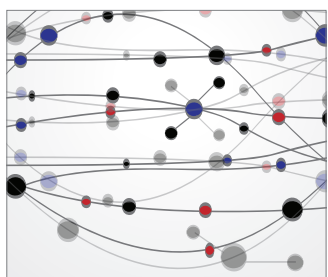

The Scientific World Journal
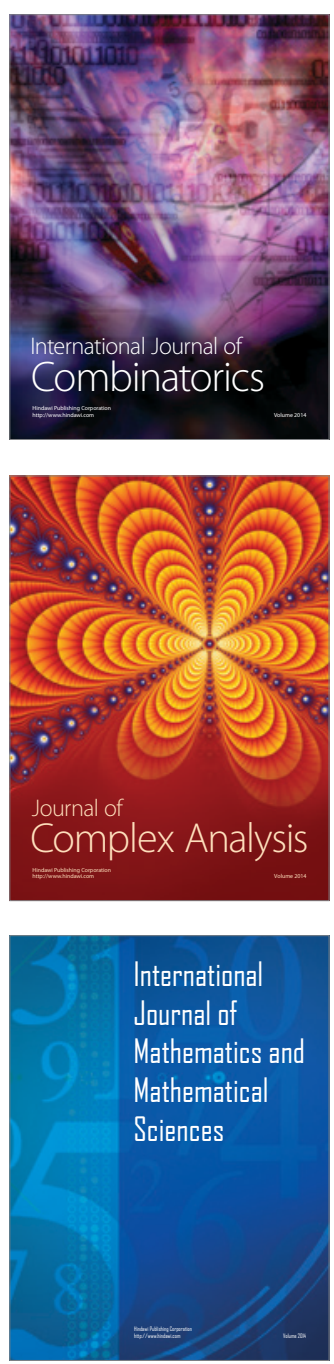
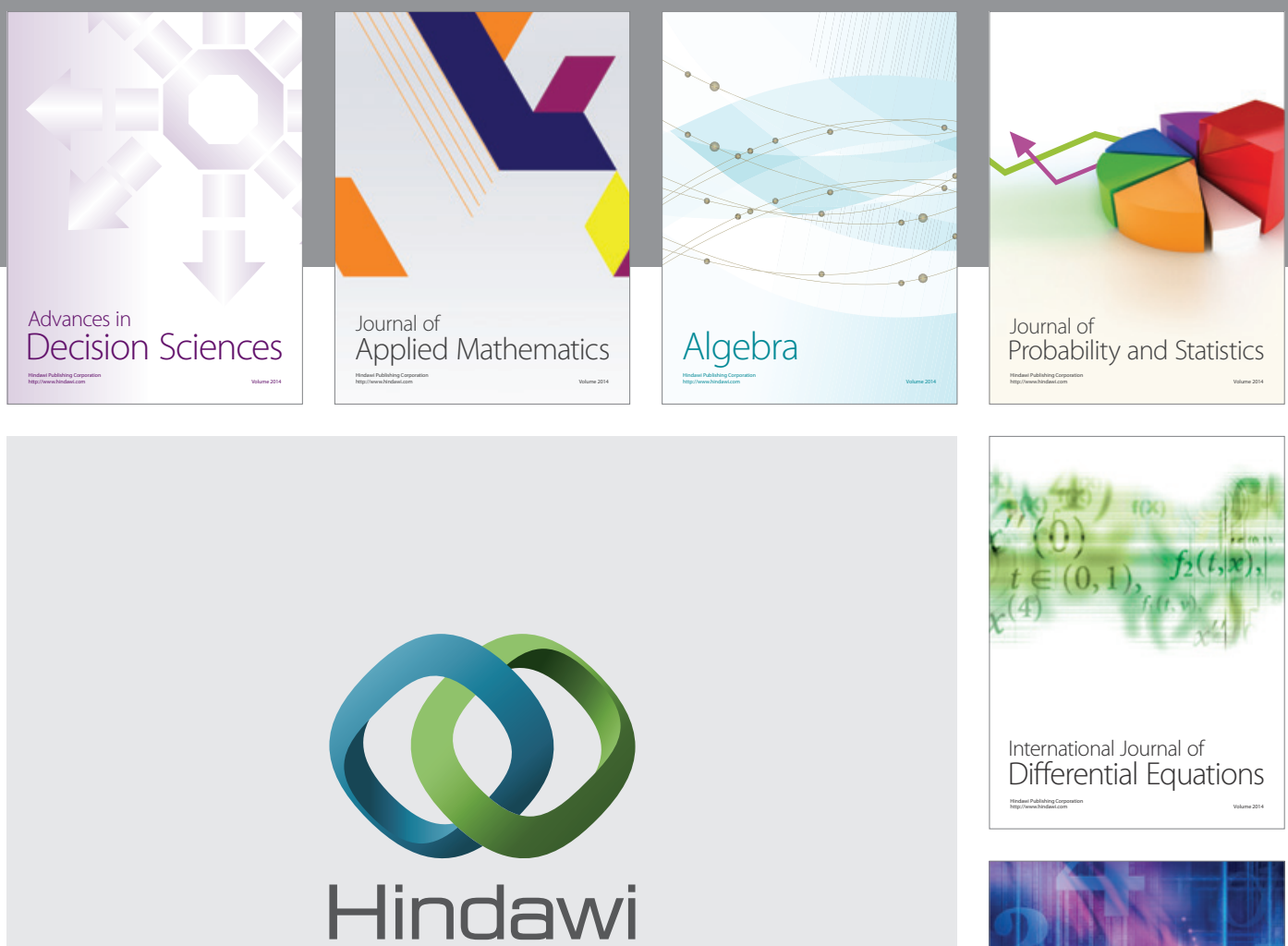

Submit your manuscripts at http://www.hindawi.com
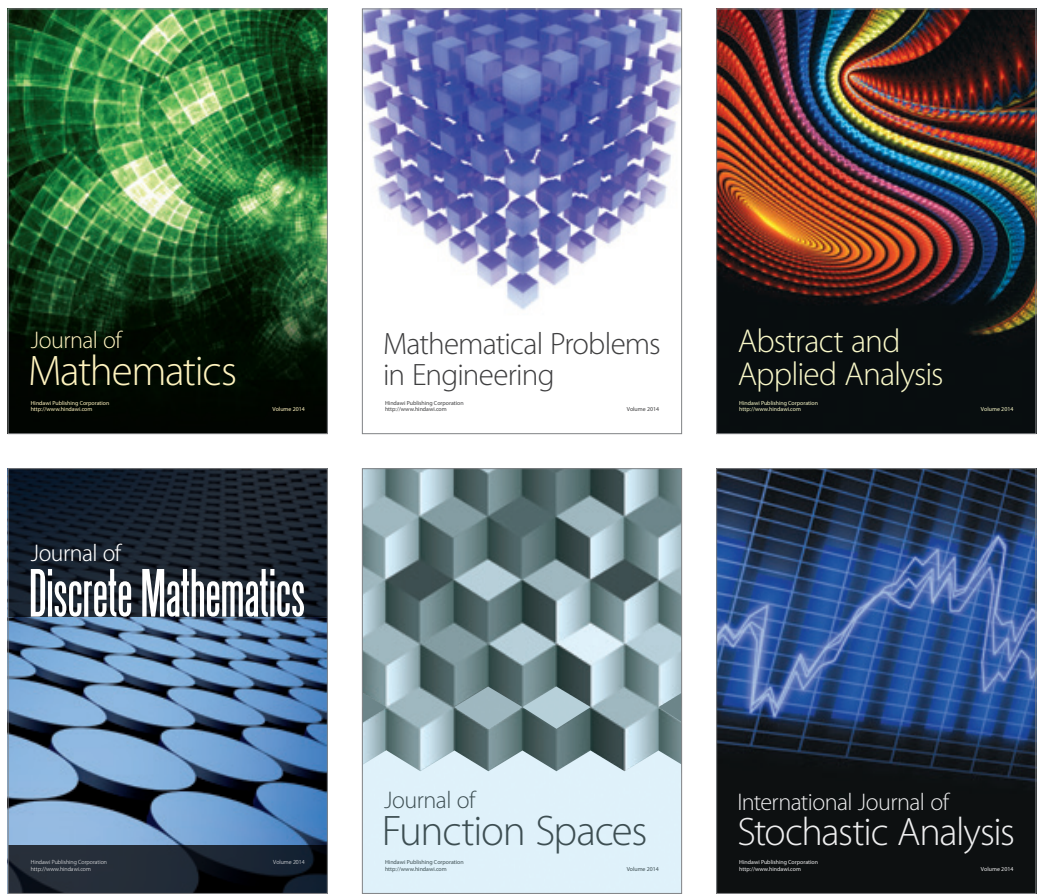

Journal of

Function Spaces

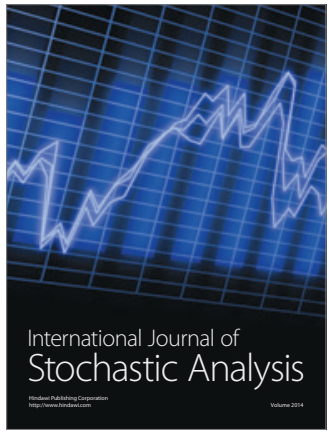

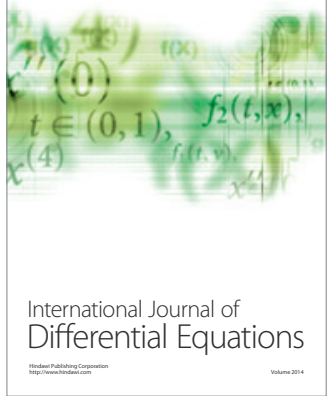
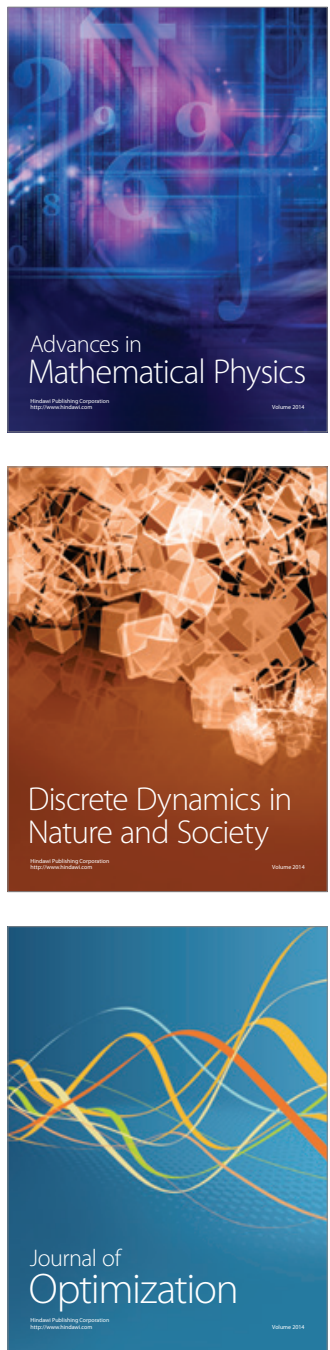